\title{
THE BAUM-CONNES CONJECTURE, NONCOMMUTATIVE POINCARÉ DUALITY, AND THE BOUNDARY OF THE FREE GROUP
}

\author{
HEATH EMERSON
}

Received 17 September 2002

\begin{abstract}
For every hyperbolic group $\Gamma$ with Gromov boundary $\partial \Gamma$, one can form the cross product $C^{*}$-algebra $C(\partial \Gamma) \rtimes \Gamma$. For each such algebra, we construct a canonical $K$-homology class. This class induces a Poincaré duality map $K_{*}(C(\partial \Gamma) \rtimes \Gamma) \rightarrow$ $K^{*+1}(C(\partial \Gamma) \rtimes \Gamma)$. We show that this map is an isomorphism in the case of $\Gamma=\mathbb{F}_{2}$, the free group on two generators. We point out a direct connection between our constructions and the Baum-Connes conjecture and eventually use the latter to deduce our result.
\end{abstract}

2000 Mathematics Subject Classification: 46L80.

1. Introduction. The aim of this paper is to point out a connection between the Baum-Connes conjecture with coefficients for the free group $\mathbb{F}_{2}$ on two generators and a Poincaré duality result for the "noncommutative space" $\partial \mathbb{F}_{2} / \mathbb{F}_{2}$, where $\partial \mathbb{F}_{2}$ is the Gromov boundary of $\mathbb{F}_{2}$, acted upon minimally by $\mathbb{F}_{2}$ through homeomorphisms.

In order to formulate what Poincaré duality should mean for a noncommutative space such as $\partial \mathbb{F}_{2} / \mathbb{F}_{2}$, one passes to the $C^{*}$-algebra cross product $C\left(\partial \mathbb{F}_{2}\right) \rtimes \mathbb{F}_{2}$ and to $K$-theory and $K$-homology for $C^{*}$-algebras. A Poincaré duality for $\partial \mathbb{F}_{2} / \mathbb{F}_{2}$ then means an isomorphism between the $K$-theory and $K$ homology of $C\left(\partial \mathbb{F}_{2}\right) \rtimes \mathbb{F}_{2}$, induced by cap product with a fixed $K$-homology class.

More generally, one can speak of $C^{*}$-algebras having Poincaré duality, or, as we call them in this paper, Poincaré duality algebras. It seems that such algebras are in some sense noncommutative analogs of $\operatorname{spin}^{c}$ manifolds. For it is well known that if $M$ is a compact $\operatorname{spin}^{c}$ manifold, the $C^{*}$-algebra $C(M)$ of continuous functions on $M$ is a Poincaré duality algebra. Such a manifold has, corresponding to the $\operatorname{spin}^{c}$ structure, a canonical elliptic operator on it-the Dirac operator-and thus (see, e.g., [9]) a canonical $K$-homology class. The cap product with this class induces the Poincaré duality isomorphism.

Various noncommutative examples of Poincaré duality $C^{*}$-algebras have been produced by Connes [3]; the first of which was the irrational rotation algebra $A_{\theta}$. Several other examples now exist, but all have the same character insofar as they are in some sense deformations of actual $\operatorname{spin}^{c}$ manifolds. 
Our example is somewhat different. The geometric data underlying $\partial \mathbb{F}_{2} / \mathbb{F}_{2}$ is highly singular: the space $\partial \mathbb{F}_{2}$ is not a homology manifold, and the group $\mathbb{F}_{2}$ is not a Poincaré duality group. It turns out to be true, however, that in factoring the space by the action of the group, that is, by forming the cross product $C^{*}$-algebra $C\left(\partial \mathbb{F}_{2}\right) \rtimes \mathbb{F}_{2}$, the resulting noncommutative space satisfies Poincaré duality.

A part of our goal is thus to point out this example and also to place it in its proper context: that of hyperbolic groups acting on their Gromov boundaries. The second part is to show, as mentioned above, a connection between our constructions and the Baum-Connes conjecture for $\mathbb{F}_{2}$.

We begin by constructing-in the full generality of hyperbolic groups-the $K$-homology class cap product with which we will induce our Poincaré duality isomorphism. It turns out that with Gromov hyperbolic groups $\Gamma$, in general there is a certain duality between functions continuous on the Gromov boundary $\partial \Gamma$ of $\Gamma$ and right translation operators on $l^{2} \Gamma$. Using this duality, we produce an algebra homomorphism $C(\partial \Gamma) \rtimes \Gamma \otimes C(\partial \Gamma) \rtimes \Gamma \rightarrow 2\left(l^{2} \Gamma\right)$, where $2\left(l^{2} \Gamma\right)=\mathscr{B}\left(l^{2} \Gamma\right) / \mathscr{K}\left(l^{2} \Gamma\right)$ denotes the Calkin algebra of $l^{2} \Gamma$, and where $\Gamma$ is an arbitrary hyperbolic group. Since $C(\partial \Gamma) \rtimes \Gamma$ is nuclear [1], such an algebra homomorphism yields via the Stinespring construction a class $\Delta \in K K^{1}(C(\partial \Gamma) \rtimes \Gamma \otimes$ $C(\partial \Gamma) \rtimes \Gamma, \mathbb{C})$, that is, a class $\Delta$ in the $K$-homology of $C(\partial \Gamma) \rtimes \Gamma \otimes C(\partial \Gamma) \rtimes \Gamma$. Kasparov product with $\Delta$ gives the required "cap-product" map $\cap \Delta: K_{*}(C(\partial \Gamma) \rtimes$ $\Gamma) \rightarrow K^{*+1}(C(\partial \Gamma) \rtimes \Gamma)$.

We next wish to prove that a cap product with $\Delta$ as above gives an isomorphism in the case of $\Gamma=\mathbb{F}_{2}$, the general case of hyperbolic groups being beyond the scope of this paper. To this end, we observe that a sort of geodesic flow on the Cayley graph of $\mathbb{F}_{2}$ may be used to construct a dual element to $\Delta$, this time in the $K$-theory of $C\left(\partial \mathbb{F}_{2}\right) \rtimes \mathbb{F}_{2} \otimes C\left(\partial \mathbb{F}_{2}\right) \rtimes \mathbb{F}_{2}$, playing the same role in this context as does the Thom class of the normal bundle of $M$ in $M \times M$ in the commutative setting. We obtain a putative inverse map $K^{*}\left(C\left(\partial \mathbb{F}_{2}\right) \rtimes \mathbb{F}_{2}\right) \rightarrow K_{*+1}\left(C\left(\partial \mathbb{F}_{2}\right) \rtimes \mathbb{F}_{2}\right)$.

We then set about calculating the composition of these two maps. The connection with the Baum-Connes conjecture appears in that the composition $K_{*}\left(C\left(\partial \mathbb{F}_{2}\right) \rtimes \mathbb{F}_{2}\right) \rightarrow K_{*}\left(C\left(\partial \mathbb{F}_{2}\right) \rtimes \mathbb{F}_{2}\right)$ turns out to be multiplication by the $\gamma$ element constructed by Julg and Valette [7].

As mentioned, the construction of our fundamental class $\Delta$ makes sense for a general hyperbolic group acting on its boundary, and in fact several of our other constructions have their counterparts for arbitrary hyperbolic groups; thus for instance, it is possible by means of the work of Gromov [5] to make sense of "geodesic flow" for an arbitrary hyperbolic group. Furthermore, although the statement $\gamma=1$ for general hyperbolic groups is false due to the possible presence of property $T$, it is nevertheless true by the work of Tu [11] that $\gamma_{\partial \Gamma \rtimes \Gamma}=1_{C(\partial \Gamma)}$, where $\gamma_{\partial \Gamma \rtimes \Gamma}$ is the $\gamma$-element for the amenable groupoid $\partial \Gamma \rtimes \Gamma$ and this weaker statement is sufficient for our purposes. Nevertheless, 
the arguments for the general case, being substantially more involved, will be dealt with in a later paper. We have chosen to emphasize the free-group case for two reasons: firstly, the relationship to the Baum-Connes conjecture is extremely explicit, and secondly, the geometry of our constructions is particularly visible.

Finally, we note that our arguments tend to suggest that the phenomenon of Poincaré duality for amenable groupoid algebras constructed from boundary actions of discrete groups is relatively common. Specifically, we expect similar results for uniform lattices in semisimple Lie groups acting on their Furstenberg boundaries, and for discrete, cocompact isometry groups of affine buildings acting on the boundaries of these buildings. Along these lines, we draw the attention of the reader to the work of Kaminker and Putnam on Cuntz-Krieger algebras (see [8]); indeed, our result (in the case of the free group of two generators) can be deduced from theirs. In fact, our work was partly motivated by the idea of finding a geometric explanation for theirs.

2. Geometric preliminaries. In this section, we work on the generality of a Gromov hyperbolic group $\Gamma$ (see [4] or [5]). So, let $\Gamma$ be such. Thus, we have fixed a generating set $S$ for $\Gamma$ and the corresponding metric $d\left(\gamma_{1}, \gamma_{2}\right)=\left|\gamma_{1}^{-1} \gamma_{2}\right|$, where $|\cdot|$ denotes the word length of a group element with respect to $S$, and with this metric, $\Gamma$ is hyperbolic in the sense of Gromov as a metric space. Note that the metric is clearly invariant under left translation.

Recall that with the hypothesis of hyperbolicity, the group $\Gamma$ viewed as a metric space can be compactified by addition of a boundary; thus, there exists a compact metrizable space $\bar{\Gamma}=\Gamma \cup \partial \Gamma$ such that $\Gamma$ sits densely in $\bar{\Gamma}$ and $\bar{\Gamma}$ is compact. The compactification is $\Gamma$-equivariant in the sense that the left translation action of $\Gamma$ extends to an action by homeomorphisms on $\bar{\Gamma}$.

There turns out to be an interesting duality between functions on $\Gamma$ which extend continuously to the Gromov compactification $\bar{\Gamma}$, and a certain class of operators on $l^{2} \Gamma$ as follows. First, we recall a definition. For what follows, let $e_{x}, e_{y}$, and so forth denote the standard basis vectors in $l^{2} \Gamma$ corresponding to points $x, y \in \Gamma$. Also, if $\tilde{f}$ is a function on $\Gamma$, we will denote by $M_{\tilde{f}}$ the corresponding multiplication operator on $l^{2} \Gamma$.

DEFINITION 2.1. An operator $T \in \mathscr{B}\left(l^{2} \Gamma\right)$ has finite propagation if there exists $R>0$ such that $\left\langle T\left(e_{x}\right), e_{y}\right\rangle=0$ whenever $d(x, y) \geq R$.

The duality we have alluded to is stated in the following lemma.

LEMMA 2.2. If $\tilde{f}$ is a function on $\Gamma$ which extends continuously to $\bar{\Gamma}$, then $\left[M_{\tilde{f}}, T\right]$ is a compact operator for all finite propagation operators $T$ on $l^{2} \Gamma$.

For the proof, we need to use the following fact about the Gromov compactification of a hyperbolic group (see [4]): note that here and elsewhere in this paper, $B_{r}(x)$, for $r>0$ and $x \in \Gamma$, denotes the ball of word-metric radius $r$ centered at $x$. 
LEMMA 2.3. If $\tilde{f}$ is a continuous function on $\bar{\Gamma}$, then for every $R>0$,

$$
\lim _{x \rightarrow \infty} \sup \left\{|f(x)-f(y)| \mid y \in B_{R}(x)\right\}=0 .
$$

Proof OF Lemma 2.2. Let $T$ be a finite propagation operator with propagation $R$ and $\tilde{f}$ be a bounded function on $\Gamma$ which extends continuously to $\bar{\Gamma}$. Then

$$
\left[M_{\tilde{f}}, T\right]\left(e_{x}\right)=\sum_{y \in B_{R}(x)}(\tilde{f}(x)-\tilde{f}(y)) T_{x y} e_{y}
$$

where $T_{x y}$ denotes as usual $\left\langle T\left(e_{x}\right), e_{y}\right\rangle$. Therefore, $\left\langle\left[M_{\tilde{f}}, T\right]\left(e_{x}\right), e_{y}\right\rangle=0$ if $d(x, y) \geq R$, and equals $(\tilde{f}(x)-\tilde{f}(y)) T_{x y}$ else. The result follows immediately from Lemma 2.3.

Let $\gamma \in \Gamma$, and let $\rho(\gamma)$ denote the unitary $l^{2} \Gamma \rightarrow l^{2} \Gamma$ induced from right translation by $\gamma, \rho(\gamma) e_{x}=e_{x \gamma^{-1}}$. The relevance of the above remarks to us lies in the following observation.

LEMMA 2.4. If $\gamma \in \Gamma$, then $\rho(\gamma)$ has a finite propagation.

Proof. One has $d\left(x, x \gamma^{-1}\right) \leq|\gamma|$ from which the result follows with $R=|\gamma|$.

COROLLARY 2.5. If $\gamma \in \Gamma$ and $\tilde{f}$ is a function on $\Gamma$ which extends continuously to $\bar{\Gamma}$, then $\left[\rho(\gamma), M_{\tilde{f}}\right]$ is a compact operator.

Now, consider the unitary $I: l^{2} \Gamma \rightarrow l^{2} \Gamma$ induced from inversion $\iota: \Gamma \rightarrow \Gamma$. Then $I \rho(\gamma) I=\lambda(\gamma)$, where $\lambda(\gamma)$ denotes left translation by $\gamma$, and $I M_{\tilde{f}} I=M_{\tilde{f} \circ \iota}$. The following corollary follows from conjugating the commutator expression appearing in Corollary 2.5 by the unitary $I$.

COROLLARY 2.6. The commutator $\left[\lambda(\gamma), M_{\tilde{f}_{\circ} l}\right]$ is a compact operator for every $\gamma \in \Gamma$ and $\tilde{f}$ a function on $\Gamma$ extending continuously to $\bar{\Gamma}$.

In Section 3, we show how the above constructions can be organized to produce a $K$-homology class inducing a Poincaré duality isomorphism.

3. $K K$-theoretic preliminaries. In this section, we recall some basic facts from Kasparov theory ( $K K$-theory). For further details, we refer the reader to $[2,9]$.

THE CATEgORY KK. Kasparov theory can be understood categorically (see [6]): there is a category $\mathbf{K K}$ whose objects are separable, nuclear $C^{*}$-algebras and whose morphisms $A \rightarrow B$ are the elements of $K K(A, B)$. There is a functor from the category of $C^{*}$-algebras to the category KK. If $\phi: A \rightarrow B$ is an algebra homomorphism $A \rightarrow B$, we denote its image under this functor 
as $[\phi]$. There is a composition or intersection product operation $K K(A, D) \times$ $K K(D, B) \rightarrow K K(A, B)$ which we denote by $(\alpha, \beta) \mapsto \alpha \otimes_{D} \beta$. If $\phi: A \rightarrow B$ is an algebra homomorphism and $D$ is any $C^{*}$-algebra, we thus have a map $\phi_{*}: K K(D, A) \rightarrow K K(D, B)$ given by $\alpha \mapsto \alpha \otimes_{A}[\phi]$. Similarly, we have a map $\phi^{*}: K K(B, D) \rightarrow K K(A, D)$ given by $\beta \mapsto[\phi] \otimes_{B} \beta$.

We will sometimes use the notations $\phi^{*}([\beta])$ and $[\phi] \otimes_{B} \beta$ interchangeably, as is warranted by the clarity of notation. Similarly with $\phi_{*}$.

If $D$ is a $C^{*}$-algebra, there is a natural map $K K(A, B) \rightarrow K K(A \otimes D, B \otimes D)$, $\alpha \mapsto \alpha \otimes 1_{D}$, and similarly a map $K K(A, B) \rightarrow K K(D \otimes A, D \otimes B)$.

GRADED COMMUTATIVITY. There are higher $K K$ groups $K K^{i}(A, B)$ for all $i \in \mathbb{Z}$, defined by $K K^{i}(A, B)=K K\left(A, B \otimes C_{i}\right)$, where $C_{i}$ is the $i$ th complex Clifford algebra, and one of the features of the theory is that the intersection product is graded commutative. If $A_{1}, \ldots, A_{n}$ are $C^{*}$-algebras, let $\sigma_{i j}$ denote the map

$$
A_{1} \otimes \cdots A_{i} \otimes \cdots A_{j} \otimes \cdots \otimes A_{n} \longrightarrow A_{1} \otimes \cdots A_{j} \otimes \cdots A_{i} \otimes \cdots \otimes A_{n}
$$

obtained by flipping the two factors. Then by graded commutativity, we mean the following: if $\alpha \in K K^{i}\left(A_{1}, B_{1}\right)$ and $\beta \in K K^{j}\left(A_{2}, B_{2}\right)$, then

$$
\begin{aligned}
& \left(\alpha \otimes 1_{A_{2}}\right) \otimes_{B_{1} \otimes A_{2}}\left(1_{B_{1}} \otimes \beta\right) \\
& \quad=(-1)^{i j}\left(\sigma_{12}\right)_{*} \sigma_{12}^{*}\left(\left(\beta \otimes 1_{A_{1}}\right) \otimes\left(1_{B_{2}} \otimes \alpha\right)\right) \in K K\left(A_{1} \otimes A_{2}, B_{1} \otimes B_{2}\right) .
\end{aligned}
$$

$K$-THEORY AND $K$-HOMOLOGY. For any $C^{*}$-algebra $A, K K^{i}(\mathbb{C}, A)=K_{i}(A)$ is the topological $K$-theory of $A$ and $K K^{i}(A, \mathbb{C})=K^{i}(A)$ is the $K$-homology of $A$ by definition.

DESCRIPTION OF EVEN CYCLES. Let $\mathscr{B}(\mathscr{E})$ denote bounded operators on a Hilbert module $\mathscr{E}, \mathscr{H}(\mathscr{E})$ denote compact operators, and $2(\mathscr{E})$ denote the Calkin algebra $\mathscr{B}(\mathscr{E}) / \mathscr{K}(\mathscr{E})$. The quotient map $\mathscr{B}(\mathscr{E}) \rightarrow 2(\mathscr{E})$ will always be denoted by $\pi$.

Following Kasparov [9], if $\mathscr{E}$ is a Hilbert $B$-module and $A$ acts on $\mathscr{E}$ by a homomorphism $A \rightarrow \mathscr{B}(\mathscr{E})$, we will refer to $\mathscr{E}$ as a Hilbert $(A, B)$-bimodule.

Because all the algebras in this paper are ungraded-or alternatively, have trivial grading-we can make certain simplifications in the definitions of the $K K$ groups (see [2]). With such ungraded $A$ and $B$, cycles for $K K(A, B)$ are given simply by pairs $(\mathscr{E}, F)$, where $\mathscr{E}$ is an $(A, B)$-bimodule, $F$ commutes modulo compact operators with the action of $A$, and $a\left(F^{*} F-1\right)$ and $a\left(F F^{*}-1\right)$ are compact for every $a \in A$.

DESCRIPTION OF ODD CYCLES. Cycles for $K K^{1}(A, B)$ are given by pairs $(\mathscr{E}, P)$ for which $P$ is an operator on the $(A, B)$-bimodule $\mathscr{E}$ satisfying the three conditions $[a, P], a\left(P^{2}-P\right)$, and $a\left(P-P^{*}\right)$ are compact for all $a \in A$.

Let $(\mathscr{E}, P)$ be an odd cycle. Then we obtain a homomorphism $A \rightarrow 2(\mathscr{E})$ by the formula $a \mapsto \pi(P a P)$. 
Conversely, let $\tau: A \rightarrow 2(\mathscr{E})$ be a homomorphism. Under the assumption of nuclearity of all algebras concerned, there exists a potentially larger Hilbert $B$-module $\tilde{\mathscr{E}}$, a representation of $A$ on $\tilde{\mathscr{E}}$, an isometry $U: \mathscr{E} \rightarrow \tilde{\mathscr{E}}$, and an operator $P$ on $\tilde{\mathscr{E}}$ such that $a\left(P^{2}-P\right),[a, P]$, and $a\left(P-P^{*}\right)$ are compact for all $a \in A$, and $\pi\left(U^{*} P a P U\right)=\tau(a)$ for all $a \in A$ (see [2]). The data $(\tilde{\mathscr{E}}, P)$ makes up an odd cycle. The process of constructing a $\tilde{\mathscr{E}}, U$, and $P$, from an extension, we will refer to as the Stinespring construction.

As a consequence, for $A$ and $B$ nuclear, we may regard $K K^{1}(A, B)$ as given by classes of maps $\tau: A \rightarrow 2(\mathscr{E})$, where $\mathscr{E}$ is a right Hilbert $B$-module. This description of $K K^{1}$-classes will be particularly appropriate to our purposes.

BOTT PERIODICITY. Recall that $K K^{-1}\left(\mathbb{C}, C_{0}(\mathbb{R})\right) \cong \mathbb{Z}$ and is generated by the class $\left[\hat{d}_{\mathbb{R}}\right]$ of the multiplier $f(x)=x / \sqrt{1+x^{2}}$ of $C_{0}(\mathbb{R})$, suitably interpreted in terms of the Clifford gradings. The class $\left[\hat{d}_{\mathbb{R}}\right]$ allows us to identify, for any $C^{*}$-algebras $A$ and $B$, the groups $K K^{1}\left(C_{0}(\mathbb{R}) \otimes A, B\right)$ and $K K(A, B)$ by the map $K K^{1}\left(C_{0}(\mathbb{R}) \otimes A, B\right) \rightarrow K K(A, B), x \mapsto\left[\hat{d}_{\mathbb{R}}\right] \otimes_{C^{*}(\mathbb{R})} x$. We will need to compute this map at the level of cycles in several simple cases.

Let $\psi$ be the function $\psi(t)=-2 i /(t+i)$ in $C_{0}(\mathbb{R})$; it has the property that $\psi+1$ is unitary in $C_{0}(\mathbb{R})^{+}$. We begin by stating the simplest version of what we will need.

LEMMA 3.1. Let $\mathrm{T}$ be a homomorphism $C_{0}(\mathbb{R}) \rightarrow 2(H)$ to the Calkin algebra of some Hilbert space $H$. Let $[\tau]$ denote the class in $K K^{1}\left(C_{0}(\mathbb{R}), \mathbb{C}\right)$ corresponding to $\tau$. Then the class $\left[\hat{d}_{\mathbb{R}}\right] \otimes_{C_{0}(\mathbb{R})}[\tau] \in K K(\mathbb{C}, \mathbb{C})$ is represented by the cycle $(H, U+1)$, where $U$ is any operator on $H$ such that $\pi(U)=\tau(\psi)$.

The significance of this simple lemma is that in the given setting, it is not necessary to explicitly represent $[\tau]$ as a $K K$-cycle (i.e., perform the Stinespring construction) in order to calculate the Kasparov product of $\left[\hat{d}_{\mathbb{R}}\right]$ and $[\tau]$. This is true also for the situation in the following lemma, which will be of direct use to us.

LEMMA 3.2. Let $A_{1}$ and $A_{2}$ be $C^{*}$-algebras and $\mathscr{E}$ be a right Hilbert $A_{2}$ module. Let $h$ be a homomorphism $C_{0}(\mathbb{R}) \otimes A_{1} \rightarrow Q(\mathscr{E})$ and $[h]$ be its class, regarded as an element of $K K^{1}\left(C_{0}(\mathbb{R}) \otimes A_{1}, A_{2}\right)$. Assume that $h$ has the form $x \otimes a_{1} \mapsto h^{\prime}(x) h^{\prime \prime}\left(a_{1}\right)$, where $h^{\prime}$ and $h^{\prime \prime}$ are homomorphisms. Suppose that the homomorphism $h^{\prime \prime}$ lifts to a homomorphism $\tilde{h}^{\prime \prime}: A_{1} \rightarrow \mathscr{B}(\mathscr{E})$. Then it follows that the class $\left[\hat{d}_{\mathbb{R}}\right] \otimes_{C_{0}(\mathbb{R})}[h] \in K K\left(A_{1}, A_{2}\right)$ is represented by the following cycle. The module is $\mathscr{E}$ with its original right $A_{2}$-module structure and the left $A_{1}$-module structure given by the homomorphism $\tilde{h}^{\prime \prime}$. The operator is given by $U+1$, where $U$ is any operator on $\mathscr{E}$ such that $\pi(U)=h^{\prime}(\psi)$.

The proof of both lemmas involves an application of the axioms for the intersection product, and is omitted (see [9]).

EQUIVARIANT $K K$. If $\Gamma$ is a group acting on $C^{*}$-algebras $A$ and $B$, we have, in addition to the group $K K(A, B)$, an equivariant group $K K_{\Gamma}(A, B)$. We will 
discuss this group briefly in connection with the $\gamma$-element and the work of Julg and Valette. It is sufficient to say that the cycles for $K K_{\Gamma}(A, B)$ consist of the same cycles as for $K K(A, B)$, but with the following extra conditions:

(1) $\Gamma$ acts as linear isometric maps on the Hilbert $(A, B)$-module $\mathscr{E}$ in such a way that $\gamma(a \xi b)=\gamma(a) \gamma(\xi) \gamma(b)$ for $a \in A, b \in B$, and $\xi \in \mathscr{E}$;

(2) the operator $F$ satisfies the condition that $\gamma(F)-F$ is compact for all $\gamma \in \Gamma$.

Regarding $\mathbf{K K}_{\Gamma}$ as a category in its own right, with morphisms $A \rightarrow B$ the elements of $K K_{\Gamma}(A, B)$, and objects $\Gamma-C^{*}$-algebras, there is a functor $\lambda: K K_{\Gamma}(A, B)$ $\rightarrow K K(A \rtimes \Gamma, B \rtimes \Gamma)$ called descent. The map $\lambda: K K_{\Gamma}(A, B) \rightarrow K K(A \rtimes \Gamma, B \rtimes \Gamma)$ can be explicitly calculated on cycles; the formulas are given in [9]. Since $\lambda$ is a functor, it takes the unit $1_{A} \in K K_{\Gamma}(A, A)$ to the unit $1_{A \rtimes \Gamma} \in K K(A \rtimes \Gamma, A \rtimes \Gamma)$, which is a fact we will make use of.

4. Construction of the fundamental class. For this section, we will return to the generality of a hyperbolic group $\Gamma$. Since $\Gamma$ acts by homeomorphisms on $\partial \Gamma$, we can consider the cross product $C^{*}$-algebra $C(\partial \Gamma) \rtimes \Gamma$, which is our main object of interest in this paper. Note that the cross product we are referring to is the reduced cross product; however, by the proof of the following lemma (whose proof can be found in [1]), the reduced and max cross products are in fact the same.

LEMMA 4.1. The algebra $C(\partial \Gamma) \rtimes \Gamma$ is nuclear and separable.

Our goal is to construct an element of the $K$-homology of the algebra $C(\partial \Gamma) \rtimes$ $\Gamma \otimes C(\partial \Gamma) \rtimes \Gamma$; specifically, an element of $K K^{1}(C(\partial \Gamma) \rtimes \Gamma \otimes C(\partial \Gamma) \rtimes \Gamma, \mathbb{C})$. This element will be presented as an extension, that is, as a map $C(\partial \Gamma) \rtimes \Gamma \otimes C(\partial \Gamma) \rtimes$ $\Gamma \rightarrow 2(H)$ for some Hilbert space $H$. By our remarks in the previous section and Lemma 4.1, such a map does produce a canonical class in $K K^{1}(C(\partial \Gamma) \rtimes \Gamma \otimes$ $C(\partial \Gamma) \rtimes \Gamma, \mathbb{C})$.

We construct two commuting maps $\lambda, \rho: C(\partial \Gamma) \rtimes \Gamma \rightarrow 2\left(l^{2} \Gamma\right)$. Let $f \in C(\partial \Gamma)$, and let $\tilde{f}$ denote any extension of $f$ to a continuous function on $\bar{\Gamma}$. Let $M_{\tilde{f}}$ denote as above the multiplication operator on $l^{2} \Gamma$ corresponding to $\tilde{f}$, and let $\lambda(f)$ be the image in $2\left(l^{2} \Gamma\right)$ of the operator $M_{\tilde{f}}$. Let $\lambda(\gamma)$ be the image in $2\left(l^{2} \Gamma\right)$ of the unitary $u_{\gamma}$ corresponding to left translation by $\gamma: u_{\gamma}\left(e_{x}\right)=e_{\gamma x}, x \in \Gamma$. It is easy to check that the assignments $f \rightarrow \lambda(f)$ and $\gamma \rightarrow \lambda(\gamma)$ define a covariant pair for the $C^{*}$-dynamical system $(C(\partial \Gamma), \Gamma)$, and so a homomorphism

$$
\lambda: C(\partial \Gamma) \rtimes \Gamma \longrightarrow 2\left(l^{2} \Gamma\right) .
$$

Next, define

$$
\rho: C(\partial \Gamma) \rtimes \Gamma \rightarrow 2\left(l^{2} \Gamma\right)
$$


by $\rho(a)=I \lambda(a) I$, where $I$ is as at the end of Section 2. Thus, $\rho(f)$ is the image in $2\left(l^{2} \Gamma\right)$ of the multiplication operator $M_{\tilde{f}_{\circ \ell}}$, and $\rho(\gamma)$ is the image in $2\left(l^{2} \Gamma\right)$ of right translation by $\gamma, e_{x} \mapsto e_{x \gamma^{-1}}$. The following theorem follows from Corollaries 2.5 and 2.6.

THEOREM 4.2. The homomorphisms $\lambda, \rho: C(\partial \Gamma) \rtimes \Gamma \rightarrow 2\left(l^{2} \Gamma\right)$ commute, and so define a homomorphism $C(\partial \Gamma) \rtimes \Gamma \otimes C(\partial \Gamma) \rtimes \Gamma \rightarrow 2\left(l^{2} \Gamma\right)$ by $a \otimes b \rightarrow \lambda(a) \rho(b)$.

DEFINITION 4.3. Let $\Delta \in K K^{1}(C(\partial \Gamma) \rtimes \Gamma \otimes C(\partial \Gamma) \rtimes \Gamma, \mathbb{C})$ denote the class corresponding to the above homomorphism $C(\partial \Gamma) \rtimes \Gamma \otimes C(\partial \Gamma) \rtimes \Gamma \rightarrow 2\left(l^{2} \Gamma\right)$.

We will refer to the class $\Delta$ as the fundamental class of the algebra $C(\partial \Gamma) \rtimes \Gamma$. Before proceeding, we note the following: let $\sigma_{12}: C(\partial \Gamma) \rtimes \Gamma \otimes C(\partial \Gamma) \rtimes \Gamma \rightarrow$ $C(\partial \Gamma) \rtimes \Gamma \otimes C(\partial \Gamma) \rtimes \Gamma$ be the homomorphism which interchanges factors and let $\sigma_{12}^{*}: K K^{1}(C(\partial \Gamma) \rtimes \Gamma \otimes C(\partial \Gamma) \rtimes \Gamma, \mathbb{C}) \rightarrow K K^{1}(C(\partial \Gamma) \rtimes \Gamma \otimes C(\partial \Gamma) \rtimes \Gamma, \mathbb{C})$ be the corresponding homomorphism of $K K$ groups. The following rather simple observation reflects a common property of "fundamental classes," that is, those classes implementing by cap product Poincaré duality isomorphisms; the author knows of no case, either commutative or not, where the fundamental class does not have it.

LEMMA 4.4. The class $\Delta$ satisfies $\sigma_{12}^{*}(\Delta)=\Delta$.

Proof. For $\sigma_{12}^{*}(\Delta)$ is the class corresponding to the map $C(\partial \Gamma) \rtimes \Gamma \otimes C(\partial \Gamma) \rtimes$ $\Gamma \rightarrow 2\left(l^{2} \Gamma\right), a \otimes b \mapsto \rho(a) \lambda(b)$. But this is unitarily conjugate to the map $a \otimes b \mapsto$ $\lambda(a) \rho(b)$ via the symmetry $I$.

We can now define the "cap-product map" interchanging the $K$-theory and $K$-homology of $C(\partial \Gamma) \rtimes \Gamma$, which we are going to show is an isomorphism when $\Gamma=\mathbb{F}_{2}$. Specifically, define

$$
\cap \Delta: K_{*}(C(\partial \Gamma) \rtimes \Gamma) \longrightarrow K^{*+1}(C(\partial \Gamma) \rtimes \Gamma)
$$

by the formula

$$
x \longmapsto\left(x \otimes 1_{C(\partial \Gamma) \rtimes \Gamma)}\right) \otimes_{C(\partial \Gamma) \rtimes \Gamma \otimes C(\partial \Gamma) \rtimes \Gamma} \Delta .
$$

Our main theorem is as follows.

THEOREM 4.5. For $\Gamma=\mathbb{F}_{2}$ and $\Delta$ as in Definition 4.3, the map $\cap \Delta$ is an isomorphism.

5. Connes' notion of Poincaré duality. In order to prove that the map $\cap \Delta$ of the previous section is an isomorphism, we will use some ideas due to Connes.

THEOREM 5.1. Let $A$ be a separable, nuclear $C^{*}$-algebra and $\Delta$ be a class in $K K^{i}(A \otimes A, \mathbb{C})$. Suppose that there exists a class $\hat{\Delta} \in K K^{-i}(\mathbb{C}, A \otimes A)$ such that 
the following equations hold:

$$
\begin{aligned}
\left(\hat{\Delta} \otimes 1_{A}\right) \otimes_{A \otimes A \otimes A}\left(1_{A} \otimes \sigma_{12}^{*} \Delta\right) & =1_{A}, \\
\left(\left(\sigma_{12}\right)_{*} \hat{\Delta} \otimes 1_{A}\right) \otimes_{A \otimes A \otimes A}\left(1_{A} \otimes \Delta\right) & =(-1)^{i} 1_{A} .
\end{aligned}
$$

Then the map

$$
\cap \Delta: K_{j}(A) \longrightarrow K^{j+i}(A)
$$

defined previously is an isomorphism with inversing (up to sign) the map $K^{j}(A) \rightarrow K_{j-i}(A)$,

$$
y \longmapsto \hat{\Delta} \otimes_{A \otimes A}\left(1_{A} \otimes y\right) .
$$

If $A$ is as above, with classes $\Delta$ and $\hat{\Delta}$ satisfying (5.1) and (5.2), respectively, we will call $A$ a Poincaré duality algebra.

Proof. The hypotheses imply the two equations

$$
\begin{aligned}
\left(\hat{\Delta} \otimes 1_{A}\right) \otimes_{A \otimes A \otimes A}\left(1_{A} \otimes \sigma_{12}^{*}(\Delta)\right) & =1_{A}, \\
\left(\left(\sigma_{12}\right)_{*}(\hat{\Delta}) \otimes 1_{A}\right) \otimes_{A \otimes A \otimes A}\left(1_{A} \otimes \Delta\right) & =(-1)^{i} 1_{A} .
\end{aligned}
$$

We show that as a consequence of these two equations,

$$
\hat{\Delta} \otimes_{A \otimes A}\left(1_{A} \otimes(y \cap \Delta)\right)=(-1)^{i j} y, \quad y \in K K^{j}(\mathbb{C}, A) .
$$

Expanding the product involved in (5.6), we obtain

$$
\hat{\Delta} \otimes_{A \otimes A}\left(1_{A} \otimes y \otimes 1_{A}\right) \otimes_{A \otimes A \otimes A}\left(1_{A} \otimes \Delta\right) .
$$

Consider the term $\left(1_{A} \otimes y \otimes 1_{A}\right) \otimes_{A \otimes A \otimes A}\left(1_{A} \otimes \Delta\right)$. It is easy to check that this is the same as $\left(1_{A} \otimes 1_{A} \otimes y\right) \otimes_{A \otimes A \otimes A}\left(1_{A} \otimes \sigma_{12}^{*}(\Delta)\right)$. Returning to the original product (5.6), we see that the latter can be written as

$$
\left(\hat{\Delta} \otimes_{A \otimes A}\left(1_{A \otimes A} \otimes y\right)\right) \otimes_{A \otimes A \otimes A}\left(1_{A} \otimes \sigma_{12}^{*}(\Delta)\right) .
$$

Now, by skew-commutativity of the external tensor product,

$$
\begin{aligned}
\hat{\Delta} \otimes_{A \otimes A}\left(1_{A \otimes A} \otimes y\right) & =(-1)^{i j}\left(\sigma_{23}\right)_{*}\left(\sigma_{12}\right)_{*}\left(y \otimes_{A}\left(1_{A} \otimes \hat{\Delta}\right)\right) \\
& =(-1)^{i j} y \otimes_{A}\left(\sigma_{23}\right)_{*}\left(\sigma_{12}\right)_{*}\left(1_{A} \otimes \hat{\Delta}\right) .
\end{aligned}
$$

Furthermore, $\left(\sigma_{23}\right)_{*}\left(\sigma_{12}\right)_{*}\left(1_{A} \otimes \hat{\Delta}\right)=\hat{\Delta} \otimes 1_{A}$. Hence, putting back into the main product, we see that (5.6) can be written as

$$
(-1)^{i j} y \otimes_{A}\left(\left(\hat{\Delta} \otimes 1_{A}\right) \otimes_{A \otimes A \otimes A}\left(1_{A} \otimes \sigma_{12}^{*}(\Delta)\right)\right)=(-1)^{i j} y,
$$

where the last equality follows from (5.1). 
REMARK 5.2. We note that if we happen to have $\Delta$ and $\hat{\Delta}$ as above, and

$$
\sigma_{12}^{*}(\Delta)=\Delta, \quad\left(\sigma_{12}\right)_{*}(\hat{\Delta})=(-1)^{i} \hat{\Delta},
$$

then the two equations (5.1) and (5.2) would be the same, and it would suffice to show that one of them holds. This is the case in the commutative setting of a compact $\operatorname{spin}^{c}$ manifold and will be the case for us also, part of which we have already proven (Lemma 4.4).

We now set about proving Theorem 4.5 in the case of $\Gamma=\mathbb{F}_{2}$ by verifying (5.1) and (5.2) of Theorem 5.1, with $A=C\left(\partial \mathbb{F}_{2}\right) \rtimes \mathbb{F}_{2}$ and $\Delta$ the fundamental class of Definition 4.3. We need first produce an element $\hat{\Delta} \in K K^{-1}\left(\mathbb{C}, C\left(\partial \mathbb{F}_{2}\right) \rtimes\right.$ $\left.\mathbb{F}_{2} \otimes C\left(\partial \mathbb{F}_{2}\right) \rtimes \mathbb{F}_{2}\right)$ playing the role of the dual element in Theorem 5.1. We will then verify (5.1), the other being rendered superfluous as a consequence of Remark 5.2, which is applicable in this case.

It will turn out, rather surprisingly, that (5.1) can be shown to be equivalent to the equation

$$
\gamma_{\partial \mathbb{F}_{2} \rtimes \mathbb{F}_{2}}=1_{C\left(\partial \mathbb{F}_{2}\right)},
$$

where $\gamma \partial \mathbb{F}_{2} \rtimes \mathbb{F}_{2}$ is the $\gamma$-element for the groupoid $\partial \mathbb{F}_{2} \rtimes \mathbb{F}_{2}$. Since this latter equation has been established by Julg and Valette, and also by Tu, we will, by this device, that is, by means of the Baum-Connes conjecture, be done.

6. Construction of a dual element. In this section, as for the rest of this paper, we specialize to the free group $\mathbb{F}_{2}$ on two generators. We are going to define an element $\hat{\Delta} \in K K^{-1}\left(\mathbb{C}, C\left(\partial \mathbb{F}_{2}\right) \rtimes \mathbb{F}_{2} \otimes C\left(\partial \mathbb{F}_{2}\right) \rtimes \mathbb{F}_{2}\right)$ serving as an "inverse" to $\Delta$.

The class $\hat{\Delta}$ will be constructed by use of the fact that any two points of $\partial \mathbb{F}_{2}$ may be connected by a unique geodesic.

By "geodesic," we mean an isometric map $r: \mathbb{Z} \rightarrow \mathbb{F}_{2}$. Topologize the collection of such $r$ by means of the metric

$$
d_{G \mathbb{F}_{2}}\left(r_{1}, r_{2}\right)=\sum_{n \in \mathbb{Z}} 2^{-|n|} d\left(r_{1}(n), r_{2}(n)\right),
$$

and denote the resulting metric space by $G \mathbb{F}_{2}$ (we follow [5]). Both $\mathbb{F}_{2}$ and $\mathbb{Z}$ act freely and properly on $G \mathbb{F}_{2}$, the former by translation $(\gamma r)(n)=\gamma r(n)$ and the latter by flow $\left(g^{n} r\right)(k)=r(k-n)$. These actions commute. Note that $G \mathbb{F}_{2} / \mathbb{F}_{2}$ is compact, whereas $G \mathbb{F}_{2} / \mathbb{Z}$ may be identified with the $\mathbb{F}_{2}$-space

$$
\partial^{2} \mathbb{F}_{2}=\left\{(a, b) \in \partial \mathbb{F}_{2} \times \partial \mathbb{F}_{2} \mid a \neq b\right\} .
$$

All these observations are easy to check. As a consequence of them, the $C^{*}$ algebras $C\left(G \mathbb{F}_{2} / \mathbb{F}_{2}\right) \rtimes \mathbb{Z}$ and $C_{0}\left(\partial^{2} \mathbb{F}_{2}\right) \rtimes \mathbb{F}_{2}$ are strongly Morita equivalent (see [10]). Let $[E]$ denote the class of the strong Morita equivalence bimodule. It is an element of $K K\left(C\left(G \mathbb{F}_{2} / \mathbb{F}_{2}\right) \rtimes \mathbb{Z}, C_{0}\left(\partial^{2} \mathbb{F}_{2}\right) \rtimes \mathbb{F}_{2}\right)$. 
On the other hand, if $u$ is the generator of $\mathbb{Z} \subset C^{*}(\mathbb{Z}) \subset C\left(G \mathbb{F}_{2} / \mathbb{F}_{2}\right) \rtimes \mathbb{Z}$, we obtain a natural homomorphism $C_{0}(\mathbb{R}) \rightarrow C\left(G \mathbb{F}_{2} / \mathbb{F}_{2}\right) \rtimes \mathbb{Z}$ by the formula $\psi \mapsto$ $u-1$ where, recall, $\psi$ is a specified generator of $C_{0}(\mathbb{R})$ satisfying $\psi+1 \in C_{0}(\mathbb{R})^{+}$ is unitary.

We denote the class in $K K\left(C_{0}(\mathbb{R}), C\left(G \mathbb{F}_{2} / \mathbb{F}_{2}\right) \rtimes \mathbb{Z}\right)$ of this homomorphism by $[u-1]$.

It will be convenient for our later computations to define an auxiliary class [D], which will lie in $K K^{-1}\left(\mathbb{C}, C_{0}\left(\partial^{2} \mathbb{F}_{2}\right) \rtimes \mathbb{F}_{2}\right)$, as follows.

Definition 6.1. The class $[D] \in K K^{-1}\left(\mathbb{C}, C_{0}\left(\partial^{2} \mathbb{F}_{2}\right) \rtimes \mathbb{F}_{2}\right)$ will be defined by

$$
[D]=\left[\hat{d_{\mathbb{R}}}\right] \otimes_{C_{0}(\mathbb{R})}[u-1] \otimes_{C\left(G \mathbb{F}_{2} / \mathbb{F}_{2}\right) \rtimes \mathbb{Z}}[E] .
$$

Next, note that the cross product $C_{0}\left(\partial^{2} \mathbb{F}_{2}\right) \rtimes \mathbb{F}_{2}$ may be regarded as a subalgebra of $C\left(\partial \mathbb{F}_{2}\right) \rtimes \mathbb{F}_{2} \otimes C\left(\partial \mathbb{F}_{2}\right) \rtimes \mathbb{F}_{2}$ via the composition of inclusions

$$
\begin{aligned}
C_{0}\left(\partial^{2} \mathbb{F}_{2}\right) \rtimes \mathbb{F}_{2} \longrightarrow C\left(\partial \mathbb{F}_{2} \times \partial \mathbb{F}_{2}\right) \rtimes \mathbb{F}_{2} \\
\cong\left(C\left(\partial \mathbb{F}_{2}\right) \otimes C\left(\partial \mathbb{F}_{2}\right)\right) \rtimes \mathbb{F}_{2} \longrightarrow C\left(\partial \mathbb{F}_{2}\right) \rtimes \mathbb{F}_{2} \otimes C\left(\partial \mathbb{F}_{2}\right) \rtimes \mathbb{F}_{2} .
\end{aligned}
$$

Let $i$ denote this composition.

Our class $\hat{\Delta}$ will be defined by the following definition.

DEFINITION 6.2. Let

$$
\hat{\Delta}=[D] \otimes_{C_{0}\left(\partial^{2} \mathbb{F}_{2}\right) \rtimes \mathbb{F}_{2}}[i] \in K K^{-1}\left(\mathbb{C}, C\left(\partial \mathbb{F}_{2}\right) \rtimes \mathbb{F}_{2} \otimes C\left(\partial \mathbb{F}_{2}\right) \rtimes \mathbb{F}_{2}\right),
$$

where $\left[\hat{d}_{\mathbb{R}}\right]$ is as in Section 3 and $[u-1]$ and $[E]$ are as above.

It will be convenient to calculate more explicitly the cycle corresponding

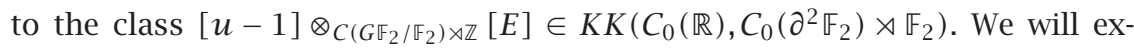
press it as a homomorphism $C_{0}(\mathbb{R}) \rightarrow C_{0}\left(\partial^{2} \mathbb{F}_{2}\right) \rtimes \mathbb{F}_{2}$, that is, as an element $w \in C_{0}\left(\partial^{2} \mathbb{F}_{2}\right) \rtimes \mathbb{F}_{2}$ such that $w+1$ is unitary in $\left(C_{0}\left(\partial^{2} \mathbb{F}_{2}\right) \rtimes \mathbb{F}_{2}\right)^{+}$.

We will first describe an element $v \in C_{0}\left(\partial^{2} \mathbb{F}_{2}\right) \rtimes \mathbb{F}_{2}$ satisfying $v^{*} v=v v^{*}=$ $\chi$, where $\chi$ is a projection. We will then set $w=v-\chi$. Then, of course, $w+1=$ $v+1-\chi$ will be unitary in $\left(C_{0}\left(\partial^{2} \mathbb{F}_{2}\right) \rtimes \mathbb{F}_{2}\right)^{+}$.

As the method of discovering such an explicit description (i.e., of transferring $K$-classes under strong Morita equivalences) is well known (see [3] in which a similar calculation is carried out in the context of $A_{\theta}$ ), we give the outcome without further discussion.

As a function on $\partial^{2} \mathbb{F}_{2} \times \mathbb{F}_{2}, v(a, b, \gamma)=1$ if and only if there exists a geodesic $r_{a, b}$ such that $r_{a, b}(-\infty)=a, r_{a, b}(+\infty)=b, r_{a, b}(0)=e$, and $r_{a, b}(-1)=\gamma$; and $v(a, b, \gamma)=0$ else.

Note that $\chi=v^{*} v=v v^{*}$ is the locally constant function on $\partial^{2} \mathbb{F}_{2}$ given by $\chi(a, b)=1$ if some (therefore any) geodesic from $a$ to $b$ passes through $e$, and equals 0 else. 
We can describe $v$ in group-algebra notation as follows. Fix $\gamma$ a generator. Then $v(\cdot, \cdot, \gamma)$ is a function on $\partial^{2} \mathbb{F}_{2}$ and, in particular, is a function on $\partial \mathbb{F}_{2} \times \partial \mathbb{F}_{2}$, whose representation as a tensor product of two functions on $\partial \mathbb{F}_{2}$ is

$$
v(\cdot, \cdot, \gamma)=\chi_{\gamma} \otimes\left(1-\chi_{\gamma}\right),
$$

where

$$
\chi_{\gamma}(a)= \begin{cases}1, & \gamma \in[e, a), \\ 0, & \text { else. }\end{cases}
$$

We can therefore represent $v$ as

$$
v=\sum_{|\gamma|=1} \chi_{\gamma} \gamma \otimes\left(1-\chi_{\gamma}\right) \gamma \in C_{0}\left(\partial^{2} \mathbb{F}_{2}\right) \rtimes \mathbb{F}_{2} \subset C\left(\partial \mathbb{F}_{2}\right) \rtimes \mathbb{F}_{2} \otimes C\left(\partial \mathbb{F}_{2}\right) \rtimes \mathbb{F}_{2} .
$$

Similarly, we represent the function $\chi$ by $\chi=\sum \chi_{\gamma} \otimes\left(1-\chi_{\gamma}\right)$, and it is easy to check that $v^{*} v=v v^{*}=\chi$ as claimed.

Finally, we note the following lemma.

LEMMA 6.3. The class $\hat{\Delta}$ satisfies $\left(\sigma_{12}\right)_{*}(\hat{\Delta})=-\hat{\Delta}$.

Proof. We have $\hat{\Delta}=i_{*}([D])$, and so

$$
\left(\sigma_{12}\right)_{*}(\hat{\Delta})=\left(\sigma_{12}\right)_{*} i_{*}([D])=\left(\sigma_{12} \circ i\right)_{*}([D])=\left(\bar{\sigma}_{12}\right)_{*}([D]),
$$

where $\bar{\sigma}_{12}: C_{0}\left(\partial^{2} \mathbb{F}_{2}\right) \rtimes \mathbb{F}_{2} \rightarrow C_{0}\left(\partial^{2} \mathbb{F}_{2}\right) \rtimes \mathbb{F}_{2}$ is the algebra homomorphism induced by the $\mathbb{F}_{2}$-equivariant map $\partial^{2} \mathbb{F}_{2} \rightarrow \partial^{2} \mathbb{F}_{2},(a, b) \mapsto(b, a)$. Now, $[D]=$ $\left[\hat{d}_{\mathbb{R}}\right] \otimes_{C_{0}(\mathbb{R})}[v-\chi]$, and hence

$$
\begin{aligned}
\left(\bar{\sigma}_{12}\right)_{*}([D]) & =\left[\hat{d}_{\mathbb{R}}\right] \otimes_{\mathcal{C}_{0}(\mathbb{R})}\left(\bar{\sigma}_{12}\right)_{*}([v-\chi]) \\
& =\left[\hat{d}_{\mathbb{R}}\right] \otimes_{\mathcal{C}_{0}(\mathbb{R})}\left[v^{*}-\chi\right]=-\left[\hat{d}_{\mathbb{R}}\right] \otimes_{\mathcal{C}_{0}(\mathbb{R})}\left[v^{*}-\chi\right]
\end{aligned}
$$

by a direct calculation, and we are done.

In the following sections, we show that in an appropriate sense, $\hat{\Delta}$ provides an "inverse" to the extension $\Delta$. More precisely, we show that the conditions of Theorem 5.1 are met by $\Delta$, the fundamental class, and the element $\hat{\Delta}$ above.

7. The $\gamma$-element. Before proceeding to verify the equations of Theorem 5.1, we need to recall the work of Julg and Valette [7].

Up to now, we have adopted the convention of writing even $K K$-cycles in the form $(\mathscr{E}, F)$, where $F$ is an operator on the module $\mathscr{E}$. A different definition is possible, in which two modules are involved, and $F$ is an operator between them. This was the setup in [7]. We retain their notation temporarily. In a moment, we describe means of geometrically describing their class in a way consistent with our conventions. 
Consider the Cayley graph $\Sigma$ for $\mathbb{F}_{2}$, which is a tree with edges $\Sigma^{1}$ and vertices $\Sigma^{0}$. Note that we work with geometric edges, that is, set-theoretic pairs of vertices $\left\{x, x^{\prime}\right\}$. If $x$ is a vertex, let $x^{\prime}$ be the vertex one unit closer to $e$, the origin, and let $s(x)$ be the edge $\left\{x, x^{\prime}\right\}$. Define an operator

$$
b: l^{2} \Sigma^{0} \longrightarrow l^{2} \Sigma^{1}
$$

by

$$
b\left(e_{x}\right)= \begin{cases}e_{s(x)}, & x \neq e \\ 0, & x=e\end{cases}
$$

Then it is clear that $b$ is an isometry, is Fredholm, and has index 1 . Next, note that $\mathbb{F}_{2}$ acts unitarily on $l^{2}\left(\Sigma^{0}\right)$ and $l^{2}\left(\Sigma^{1}\right)$, and that, furthermore, $\gamma b \gamma^{-1}-b$ is a compact (in fact finite rank) operator, for all $\gamma \in \mathbb{F}_{2}$.

It follows that the pair $\left(l^{2} \Sigma^{0} \oplus l^{2} \Sigma^{1},\left(\begin{array}{cc}0 & b^{*} \\ b & 0\end{array}\right)\right)$ defines a cycle for $K K_{\mathbb{F}_{2}}(\mathbb{C}, \mathbb{C})$.

Let $\gamma$ denote its class. That $\gamma=1$ in this group implies the Baum-Connes conjecture for $\mathbb{F}_{2}$. This fact (that $\gamma=1$ ) was proved by Julg and Valette in [7].

We can produce a cycle for $K K_{\mathbb{F}_{2}}\left(C\left(\partial \mathbb{F}_{2}\right), C\left(\partial \mathbb{F}_{2}\right)\right)$, whose class we will denote by $\gamma_{\partial \mathbb{F}_{2}}$, by tensoring all the above data with $C\left(\partial \mathbb{F}_{2}\right)$. Thus, let $\mathscr{E}^{0}=$ $C\left(\partial \mathbb{F}_{2} ; l^{2}\left(\Sigma^{0}\right)\right)$ and $\mathscr{E}^{1}=C\left(\partial \mathbb{F}_{2} ; l^{2}\left(\Sigma^{1}\right)\right)$. Let $B: \mathscr{E}^{0} \rightarrow \mathscr{E}^{1}$ be defined by $(B \xi)(a)=$ $b(\xi(a))$. The Hilbert $C\left(\partial \mathbb{F}_{2}\right)$-modules $\mathscr{E}^{i}$ carry obvious actions of $\mathbb{F}_{2}$. Let $\gamma_{\partial \mathbb{F}_{2}}$ be the class of the cycle $\left(\mathscr{E}^{0} \oplus \mathscr{E}^{1},\left(\begin{array}{cc}0 & B^{*} \\ B & 0\end{array}\right)\right)$. It is easy to check that the process of tensoring with $C\left(\partial \mathbb{F}_{2}\right)$ in this way preserves units, that is,

$$
\gamma=1 \Longrightarrow \gamma_{\partial \mathbb{F}_{2}}=1_{C\left(\partial \mathbb{F}_{2}\right)}
$$

in the ring $K K_{\mathbb{F}_{2}}\left(C\left(\partial \mathbb{F}_{2}\right), C\left(\partial \mathbb{F}_{2}\right)\right)$. Hence, we have the following lemma.

LEMMA 7.1. The cycle $\left(\mathscr{E}^{0} \oplus \mathscr{E}^{1},\left(\begin{array}{cc}0 & B^{*} \\ B & 0\end{array}\right)\right)$ is equivalent to the cycle corresponding to $1_{C\left(\partial \mathbb{F}_{2}\right)}$ in the group $K K_{\mathbb{F}_{2}}\left(C\left(\partial \mathbb{F}_{2}\right), C\left(\partial \mathbb{F}_{2}\right)\right)$.

We now set about describing a cycle equivalent to the above, but which is in some sense simpler. To do this, it will be notationally and conceptually simpler to work with fields. Thus, we note that $\mathscr{E}^{0}$ and $\mathscr{E}^{1}$ may be viewed as sections of the constant fields of Hilbert spaces $\left\{H_{a}^{0} \mid a \in \partial \mathbb{F}_{2}\right\}$ and $\left\{H_{a}^{1} \mid a \in \partial \mathbb{F}_{2}\right\}$, respectively, with $H_{a}^{0}=l^{2}\left(\Sigma^{0}\right)$ and $H_{a}^{1}=l^{2}\left(\Sigma^{1}\right)$ for all $a \in \partial \mathbb{F}_{2}$, and that the operator $B$ may be regarded as the constant family of operators $\left\{b_{a} \mid a \in \partial \mathbb{F}_{2}\right\}$ with $b_{a}=b$ for all $a \in \partial \mathbb{F}_{2}$. What we are going to do is to eliminate edges from the cycle at the expense of changing the constant field of operators to a nonconstant field.

To this end, consider the field of unitary maps $\left\{U_{a}: H_{a}^{1} \rightarrow H_{a}^{0} \mid a \in \partial \mathbb{F}_{2}\right\}$ given by $U_{a}\left(e_{s}\right)=e_{x}$, where $x$ is the vertex of $s$ farthest from $a$. Note that the assignment $a \mapsto U_{a}$, though not constant, is strongly continuous. For if $a$ and $b$ are two boundary points, then $U_{a}=U_{b}$ except for edges lying on the geodesic 
$(a, b)$. Consequently, if $s$ is a fixed edge and $a$ and $b$ are close enough, then $U_{a}\left(e_{s}\right)=U_{b}\left(e_{s}\right)$ since if $a$ and $b$ are sufficiently close, $s$ does not lie on $(a, b)$. Now, consider the composition

$$
l^{2} \mathbb{F}_{2}=H_{a}^{0} \stackrel{b_{a}}{\longrightarrow} H_{a}^{1} \stackrel{U_{a}}{\longrightarrow} H_{a}^{0}=l^{2} \mathbb{F}_{2},
$$

which we denote by $W_{a}$. We see that for $x=e, W_{a}\left(e_{x}\right)=0$, and for $x \neq e$, we have

$$
W_{a}\left(e_{x}\right)= \begin{cases}e_{x^{\prime}}, & x \in[e, a), \\ e_{x}, & \text { else, }\end{cases}
$$

where $x^{\prime}$ is the vertex one unit closer to $e$ than $x$.

Since the assignment $a \rightarrow W_{a}$ is continuous, we obtain a Hilbert $C\left(\partial \mathbb{F}_{2}\right)$ module map $\mathscr{E}^{0} \rightarrow \mathscr{E}^{0}$ by defining for $\xi \in C\left(\partial \mathbb{F}_{2} ; l^{2} \mathbb{F}_{2}\right),(W \xi)(a)=W_{a}(\xi(a))$. Then, by unitary invariance of $K K$ and the work of Julg and Valette, we see the following lemma.

LEMMA 7.2. The cycle $\left(\mathscr{E}^{0} \oplus \mathscr{E}^{0},\left(\begin{array}{cc}0 & W^{*} \\ W & 0\end{array}\right)\right)$ is equivalent to the cycle corresponding to $1_{\partial \mathbb{F}_{2}}$ in the group $K K_{\mathbb{F}_{2}}\left(C\left(\partial \mathbb{F}_{2}\right), C\left(\partial \mathbb{F}_{2}\right)\right)$.

Since we have now altered the cycle of Julg and Valette up to equivalence so that only one Hilbert module is involved (it is now otherwise known as an "evenly graded" Fredholm module), we may now return as promised to our conventions and write it simply as

$$
\left(C\left(\partial \mathbb{F}_{2} ; l^{2} \mathbb{F}_{2}\right), W\right),
$$

consistent with the way we have been writing (even) $K K$-cycles up to now.

To summarize, we have

$$
\left[\left(C\left(\partial \mathbb{F}_{2} ; l^{2} \mathbb{F}_{2}\right), W\right)\right]=\left[1_{C\left(\partial \mathbb{F}_{2}\right)}\right] \in K K_{\mathbb{F}_{2}}\left(C\left(\partial \mathbb{F}_{2}\right), C\left(\partial \mathbb{F}_{2}\right)\right) .
$$

Next, we apply the descent map

$$
\lambda: K K_{\mathbb{F}_{2}}\left(C\left(\partial \mathbb{F}_{2}\right), C\left(\partial \mathbb{F}_{2}\right)\right) \rightarrow K K\left(C\left(\partial \mathbb{F}_{2}\right) \rtimes \mathbb{F}_{2}, C\left(\partial \mathbb{F}_{2}\right) \rtimes \mathbb{F}_{2}\right)
$$

to the cycle described in equation (7.6) thus producing a cycle for $K K\left(C\left(\partial \mathbb{F}_{2}\right) \rtimes\right.$ $\left.\mathbb{F}_{2}, C\left(\partial \mathbb{F}_{2}\right) \rtimes \mathbb{F}_{2}\right)$ which, by functoriality of descent, will be equivalent to the cycle corresponding to $1_{C\left(\partial \mathbb{F}_{2}\right) \rtimes \mathbb{F}_{2}}$. A direct appeal to the definition of $\lambda$ (see [9]) produces the cycle $\left(C\left(\partial \mathbb{F}_{2}\right) \rtimes \mathbb{F}_{2} \otimes l^{2} \mathbb{F}_{2}, \bar{W}\right)$, where, regarding $C\left(\partial \mathbb{F}_{2}\right) \rtimes \mathbb{F}_{2} \otimes l^{2} \mathbb{F}_{2}$ as given by functions $\mathbb{F}_{2} \rightarrow C\left(\partial \mathbb{F}_{2}\right) \otimes l^{2} \mathbb{F}_{2}$, the action of $\bar{W}$ on these functions is given by the formula $(\bar{W} \xi)(\gamma)=W(\xi(\gamma))$. We have the following lemma.

LEMMA 7.3. The cycle $\left(C\left(\partial \mathbb{F}_{2}\right) \rtimes \mathbb{F}_{2} \otimes l^{2} \mathbb{F}_{2}, \bar{W}\right)$ is equivalent to the cycle corresponding to $1_{C\left(\partial \mathbb{F}_{2}\right) \rtimes \mathbb{F}_{2}}$ in $K K\left(C\left(\partial \mathbb{F}_{2}\right) \rtimes \mathbb{F}_{2}, C\left(\partial \mathbb{F}_{2}\right) \rtimes \mathbb{F}_{2}\right)$. 
This concludes our preparatory work. We now show that the class of the cycle given in the above lemma is the same as the class of the Kasparov product of the elements $\hat{\Delta}$ and $\Delta$, concluding thus, as a consequence of the work of Julg and Valette, that (5.1) holds.

8. Untwisting. We are interested in calculating the cycle corresponding to the Kasparov product

$$
\left(\hat{\Delta} \otimes 1_{C\left(\partial \mathbb{F}_{2}\right) \rtimes \mathbb{F}_{2}}\right) \otimes_{C\left(\partial \mathbb{F}_{2}\right) \rtimes \mathbb{F}_{2} \otimes C\left(\partial \mathbb{F}_{2}\right) \rtimes \mathbb{F}_{2} \otimes C\left(\partial \mathbb{F}_{2}\right) \rtimes \mathbb{F}_{2}}\left(1_{C\left(\partial \mathbb{F}_{2}\right) \rtimes \mathbb{F}_{2}} \otimes \sigma_{12}^{*} \Delta\right) .
$$

In this section, we do something we call-following an analogous procedure in [8] - "untwisting." A simple but fundamental property of hyperbolic groups-and in particular of the free group-will be used: specifically, if two points $a$ and $b$ on $\partial \mathbb{F}_{2}$ are sufficiently far apart, then any geodesic connecting them passes quite close to the identity e of the group. This follows immediately from the definition of the topology on the compactified space $\overline{\mathbb{F}}_{2}$. More precisely, we have the following lemma.

LEMMA 8.1. Let $\tilde{N}$ be a neighborhood of the diagonal $\left\{(a, a) \mid a \in \partial \mathbb{F}_{2}\right\}$ in $\partial \mathbb{F}_{2} \times \overline{\mathbb{F}}_{2}$. Then there exists $R>0$ such that if $(a, b) \in\left(\partial \mathbb{F}_{2} \times \overline{\mathbb{F}}_{2}\right) \backslash \tilde{N}$, then the (unique) geodesic from $a$ to $b$ passes through $B_{R}(e)$.

NoTE 8.2. To simplify the notation in this section, we denote by $A$ the cross product $C\left(\partial \mathbb{F}_{2}\right) \rtimes \mathbb{F}_{2}$ and by $B$ the algebra $C_{0}\left(\partial^{2} \mathbb{F}_{2}\right) \rtimes \mathbb{F}_{2}$.

Consider then the product $\left(\hat{\Delta} \otimes 1_{A}\right) \otimes_{A \otimes A \otimes A}\left(1_{A} \otimes \sigma_{12}^{*} \Delta\right)$ involved in the lefthand side of (5.1).

Since $\hat{\Delta}=i_{*}([D])=[D] \otimes_{B}[i]$, we have

$$
\begin{aligned}
& \left(\hat{\Delta} \otimes 1_{A}\right) \otimes_{A \otimes A \otimes A}\left(1_{A} \otimes \sigma_{12}^{*} \Delta\right) \\
& \quad=\left([D] \otimes 1_{A}\right) \otimes_{B \otimes A}\left[i \otimes 1_{A}\right] \otimes_{A \otimes A \otimes A}\left(1_{A} \otimes \sigma_{12}^{*} \Delta\right) .
\end{aligned}
$$

We begin by examining the term $\left[i \otimes 1_{A}\right] \otimes_{A \otimes A \otimes A}\left(1_{A} \otimes \sigma_{12}^{*} \Delta\right) \in K K^{1}(B \otimes$ $A, A)$. It is easy to describe the corresponding cycle explicitly. For since $\sigma_{12}^{*} \Delta$ is represented by a map $A \otimes A \rightarrow 2\left(l^{2} \mathbb{F}_{2}\right)$, so also $1_{A} \otimes \sigma_{12}^{*} \Delta$ is represented by a map $A \otimes A \otimes A \rightarrow 2\left(A \otimes l^{2} \mathbb{F}_{2}\right)$ and $\left[i \otimes 1_{A}\right] \otimes_{A \otimes A \otimes A}\left(1_{A} \otimes \sigma_{12}^{*} \Delta\right)$ is represented by a map $B \otimes A \rightarrow 2\left(A \otimes l^{2} \mathbb{F}_{2}\right)$. By construction, this map is given on the set of elementary tensors by the formula

$$
a_{1} \otimes a_{2} \otimes a_{3} \longmapsto a_{1} \otimes \rho\left(a_{2}\right) \lambda\left(a_{3}\right),
$$

where we have suppressed the inclusion $i: B \rightarrow A \otimes A$, so that $a_{1} \otimes a_{2}$ in the above expression is understood as an element of $B$.

We first show that the above map up to unitary equivalence can be rewritten in a much more tractable way.

Before proceeding, let $\tilde{G}$ be a function on $\partial \mathbb{F}_{2} \times \mathbb{F}_{2}$ not necessarily continuous in the second variable, but continuous in the first. Then $\tilde{G}$ can be made to act 
on the right $A$-module $A \otimes l^{2} \mathbb{F}_{2}$ by the formula

$$
\tilde{G} \cdot\left(a \otimes e_{y}\right)=\tilde{G}(\cdot, y) a \otimes e_{y},
$$

noting that for each $y \in \mathbb{F}_{2}, \tilde{G}(\cdot, y) \in C\left(\partial \mathbb{F}_{2}\right) \subset A$.

Now, let $F$ be a continuous, compactly supported function on $\partial^{2} \mathbb{F}_{2}$. Thus, $F$ is a continuous function on $\partial \mathbb{F}_{2} \times \partial \mathbb{F}_{2}$ vanishing in a neighborhood of the diagonal. So, we can extend it to a continuous function $\tilde{F}$ on $\partial \mathbb{F}_{2} \times \overline{\mathbb{F}}_{2}$ by the Tietze extension theorem and restrict the result to $\partial \mathbb{F}_{2} \times \mathbb{F}_{2}$. Let $\tilde{F}^{\prime}$ denote the function on $\partial \mathbb{F}_{2} \times \mathbb{F}_{2}$ given by $(a, x) \mapsto \tilde{F}\left(x^{-1} a, x^{-1}\right)$. Note that $\tilde{F}^{\prime}$ is continuous in the first variable, but not in the second. Hence, $\tilde{F}^{\prime}$ may be made to act on the Hilbert $A$-module $A \otimes l^{2} \mathbb{F}_{2}$ by the remark in the previous paragraph. We can thus regard $\tilde{F}^{\prime}$ as an element of $\mathscr{B}\left(A \otimes l^{2} \mathbb{F}_{2}\right)$. Let $\tau(F)$ denote the image of the operator $\tilde{F}^{\prime}$ in $2\left(A \otimes l^{2} \mathbb{F}_{2}\right)$.

Remark that $F \mapsto \tau(F)$ is a well-defined homomorphism $C_{0}\left(\partial^{2} \mathbb{F}_{2}\right) \rightarrow 2(A \otimes$ $\left.l^{2} \mathbb{F}_{2}\right)$. For any two extensions of $F$ to functions on $\partial \mathbb{F}_{2} \times \overline{\mathbb{F}}_{2}$ differ by a functionsay $\tilde{H}$-vanishing on $\partial \mathbb{F}_{2} \times \partial \mathbb{F}_{2}$, then $\tilde{H}^{\prime}$ also vanishes on $\partial \mathbb{F}_{2} \times \partial \mathbb{F}_{2}$, and so defines an operator lying in $\mathscr{K}\left(A \otimes l^{2} \mathbb{F}_{2}\right)$.

Next, for $\gamma \in \mathbb{F}_{2}$, set $\tau(\gamma)=1 \otimes \rho(\gamma) \in 2\left(A \otimes l^{2} \mathbb{F}_{2}\right)$. It is a routine computation to check that the assignments

$$
F \longmapsto \tau(F), \quad \gamma \longmapsto \rho(\gamma)
$$

make up a covariant pair for the dynamical system $\left(C_{0}\left(\partial^{2} \mathbb{F}_{2}\right), \mathbb{F}_{2}\right)$, and hence a homomorphism

$$
\tau: B \longrightarrow 2\left(A \otimes l^{2} \mathbb{F}_{2}\right)
$$

Next, define a covariant pair for the dynamical system $\left(C\left(\partial \mathbb{F}_{2}\right), \mathbb{F}_{2}\right)$ by $\varphi(f)=$ $f \otimes 1 \in \mathscr{B}\left(A \otimes l^{2} \mathbb{F}_{2}\right)$ and $\varphi(\gamma)=\gamma \otimes u_{\gamma} \in \mathscr{B}\left(A \otimes l^{2} \mathbb{F}_{2}\right)$. It is similarly easy to check that this makes up a covariant pair and so a homomorphism

$$
\varphi: A \longrightarrow \mathscr{B}\left(A \otimes l^{2} \mathbb{F}_{2}\right) .
$$

The following proposition is a key to the untwisting argument.

Proposition 8.3. The class $\left[i \otimes 1_{A}\right] \otimes_{A \otimes A \otimes A}\left(1_{A} \otimes \sigma_{12}^{*} \Delta\right) \in K K^{1}(B \otimes A, A)$ is represented by the homomorphism $\iota: B \otimes A \rightarrow 2\left(A \otimes l^{2} \mathbb{F}_{2}\right)$ such that

$$
\iota(b \otimes a)=\tau(b) \pi(\varphi(a)), \quad b \in B, a \in A,
$$

where $\varphi$ and $\tau$ are as above.

We note that the homomorphisms $\tau$ and $\pi \circ \varphi$ commute, and so $\iota$ actually is a homomorphism. That $\iota$ is a homomorphism also follows, however, from the proof of Proposition 8.3 below, which shows that $\iota$ is unitarily conjugate to the map in (8.3). 
We will require the following lemma.

LEMmA 8.4. Let $k \in C_{c}\left(\partial^{2} \mathbb{F}_{2} \times \partial \mathbb{F}_{2}\right)$ and $\tilde{k}$ be an extension of $k$ to a continuous function on $\partial \mathbb{F}_{2} \times \overline{\mathbb{F}}_{2} \times \overline{\mathbb{F}}_{2}$. Then the two functions on $\partial \mathbb{F}_{2} \times \mathbb{F}_{2}$

$$
\begin{aligned}
& (a, x) \longmapsto \tilde{k}\left(x^{-1}(a), x^{-1}, x\right), \\
& (a, x) \longmapsto \tilde{k}\left(x^{-1}(a), x^{-1}, a\right)
\end{aligned}
$$

are the same modulo $C_{0}\left(\partial \mathbb{F}_{2} \times \mathbb{F}_{2}\right)$.

Proof. Let $k$ be as in the statement of the lemma. Then for some neighborhood $N$ of the diagonal in $\partial \mathbb{F}_{2} \times \partial \mathbb{F}_{2}, k$ is supported on $\left(\partial \mathbb{F}_{2} \times \partial \mathbb{F}_{2} \times \partial \mathbb{F}_{2}\right) \backslash(N \times$ $\partial \mathbb{F}_{2}$ ). It follows that we can choose an extension $\tilde{k}$ of $k$ to a function on $\partial \mathbb{F}_{2} \times$ $\overline{\mathbb{F}}_{2} \times \overline{\mathbb{F}}_{2}$ such that there is a neighborhood $\tilde{N}$ of the diagonal in $\partial \mathbb{F}_{2} \times \overline{\mathbb{F}}_{2}$ such that $\tilde{k}$ is supported in $\left(\partial \mathbb{F}_{2} \times \overline{\mathbb{F}}_{2} \times \overline{\mathbb{F}}_{2}\right) \backslash\left(\tilde{N} \times \overline{\mathbb{F}}_{2}\right)$.

Now, by routine compactness arguments, it suffices to show that for $a \in \partial \mathbb{F}_{2}$ fixed and $x_{n}$ a sequence in $\mathbb{F}_{2}$ converging to a boundary point $b \in \partial \mathbb{F}_{2}$, the sequence

$$
\tilde{k}\left(x_{n}^{-1}(a), x_{n}^{-1}, x_{n}\right)-\tilde{k}\left(x_{n}^{-1}(a), x_{n}^{-1}, a\right)
$$

converges to 0 as $n \rightarrow \infty$. We may clearly also assume without loss of generality that for all $n$, the point $\left(x_{n}^{-1}(a), x_{n}^{-1}\right)$ lies in the complement of $\tilde{N}$, else both terms are 0 . By Lemma 8.1, there exists $R>0$ such that any two points $(c, z) \in \partial \mathbb{F}_{2} \times \mathbb{F}_{2}$ not in $\tilde{N}$ have the property that the geodesic $[z, c)$ passes through $B_{R}(e)$. Thus, for all $n$ large enough, $d\left(e,\left[x_{n}^{-1}, x_{n}^{-1}(a)\right) \leq R\right.$. But then $d\left(x_{n},[e, a)\right) \leq R$ for all $n$. If a sequence in a hyperbolic space remains at fixed, bounded distance from a geodesic ray, it must converge to the endpoint of the ray. Hence, $x_{n} \rightarrow a$, and we are done by continuity of $\tilde{k}$ in the third variable.

Proof of Proposition 8.3. Consider the class $\left[i \otimes 1_{A}\right] \otimes_{A \otimes A \otimes A}\left(1_{A} \otimes\right.$ $\left.\sigma_{12}^{*} \Delta\right)$, which is represented by the map $B \otimes A \rightarrow 2\left(A \otimes l^{2} \mathbb{F}_{2}\right)$ in (8.3).

Define a unitary map of Hilbert modules $U: A \otimes l^{2} \mathbb{F}_{2} \rightarrow A \otimes l^{2} \mathbb{F}_{2}$ by the formula $U\left(a \otimes e_{x}\right)=x \cdot a \otimes e_{x}$. Let $A d_{U}$ denote the inner automorphism of $2\left(A \otimes l^{2} \mathbb{F}_{2}\right)$ given by $\pi(T) \mapsto \pi\left(U T U^{*}\right)$, and let $\iota^{\prime}$ denote the homomorphism $B \otimes A \rightarrow 2\left(A \otimes l^{2} \mathbb{F}_{2}\right)$, where

$$
\iota^{\prime}\left(a_{1} \otimes a_{2} \otimes a_{3}\right)=A d_{U}\left(a_{1} \otimes \rho\left(a_{2}\right) \lambda\left(a_{3}\right)\right) .
$$

We claim that $\iota^{\prime}=\iota$. It is a simple matter to check that $\iota_{B \otimes C_{r}^{*}\left(\mathbb{F}_{2}\right)}=\iota_{\left.\right|_{B \otimes C_{r}^{*}\left(\mathbb{F}_{2}\right)} ^{\prime}}$, where $B \otimes C_{r}^{*}\left(\mathbb{F}_{2}\right)$ is viewed as a subalgebra of $B \otimes A$, and that for $b \in B$ and $f \in$ $C\left(\partial \mathbb{F}_{2}\right)$, we have $\iota(b \otimes f)=\tau(b) \pi(f \otimes 1)$ whereas $\iota^{\prime}(b \otimes f)=\tau(b)(1 \otimes \lambda(f))$. Thus, it remains to prove that $\tau(b) \pi\left(1 \otimes M_{\tilde{f}}-f \otimes 1\right)=0$ in the Calkin algebra $2\left(A \otimes l^{2} \mathbb{F}_{2}\right)$ whenever $b \in B, f \in C\left(\partial \mathbb{F}_{2}\right)$, and $\tilde{f}$ is an extension of $f$ to $\overline{\mathbb{F}}_{2}$. The collection of $b$ of the form $\sum \gamma F_{\gamma}$ with each $F_{\gamma} \in C_{c}\left(\partial^{2} \mathbb{F}_{2}\right)$ is dense in $B$, and 
hence it suffices to prove the result for $b$ having this form. Hence, it is sufficient to prove the result for $b=F \in C_{c}\left(\partial^{2} \mathbb{F}_{2}\right)$. We are now done by Lemma 8.4 with $k(a, b, c)=F(a, b) f(c)$.

9. Conclusion of the proof. Consider the class $\left[i \otimes 1_{A}\right] \otimes_{A \otimes A \otimes A}\left(1_{A} \otimes \sigma_{12}^{*} \Delta\right)$, which we have shown has the form $[\iota]$, where $\iota$ is as in Proposition 8.3. We are interested in calculating the Kasparov product of the class of this extension and the class $[D] \otimes 1_{A} \in K K^{-1}(A, B \otimes A)$.

Recall that

$$
[D]=\left[\hat{d}_{\mathbb{R}}\right] \otimes_{C_{0}(\mathbb{R})}[v-\chi],
$$

where $[v-\chi]$ is the class of the homomorphism $C_{0}(\mathbb{R}) \rightarrow B$ induced by mapping $\psi$ to $v-\chi$.

Hence, $[D] \otimes 1_{A}=\left(\left[\hat{d}_{\mathbb{R}}\right] \otimes 1_{A}\right) \otimes_{C_{0}(\mathbb{R}) \otimes A}\left([v-\chi] \otimes 1_{A}\right)$, where $[v-\chi] \otimes 1_{A}$ is represented by the homomorphism $C_{0}(\mathbb{R}) \otimes A \rightarrow B \otimes A$ induced by mapping $\psi \otimes a \mapsto(v-\chi) \otimes a$.

The Kasparov product

$$
\left([D] \otimes 1_{A}\right) \otimes_{B \otimes A}\left[i \otimes 1_{A}\right] \otimes_{A \otimes A \otimes A}\left(1_{A} \otimes \sigma_{12}^{*}(\Delta)\right)
$$

therefore has the form

$$
\left(\left[\hat{d}_{\mathbb{R}}\right] \otimes 1_{A}\right) \otimes_{C_{0}(\mathbb{R}) \otimes A}\left(\left([v-\chi] \otimes 1_{A}\right) \otimes_{B \otimes A}[\iota]\right),
$$

and $\left([v-\chi] \otimes 1_{A}\right) \otimes_{B \otimes A}[\iota]$ is represented by the homomorphism $C_{0}(\mathbb{R}) \otimes A \rightarrow$ $2\left(A \otimes l^{2} \mathbb{F}_{2}\right)$ induced by mapping

$$
\psi \otimes a \longmapsto \tau(v-\chi) \pi(\varphi(a))
$$

But, this homomorphism has the form stated in the hypothesis of Lemma 3.2. By that lemma,

$$
\left(\left[\hat{d}_{\mathbb{R}}\right] \otimes 1_{A}\right) \otimes_{C_{0}(\mathbb{R}) \otimes A}\left([v-\chi] \otimes 1_{A}\right) \otimes_{B \otimes A}[\iota]
$$

is represented by the $K K(A, A)$ cycle $\left(A \otimes l^{2} \mathbb{F}_{2}, \bar{U}+1\right)$, where $\bar{U}$ is any lift to $\mathscr{B}\left(A \otimes l^{2} \mathbb{F}_{2}\right)$ of the element $\tau(v-\chi) \in 2\left(A \otimes l^{2} \mathbb{F}_{2}\right)$, where the Hilbert $(A, A)$ bimodule $A \otimes l^{2} \mathbb{F}_{2}$ has its standard right $A$-module structure, and where it has the left $A$-module structure given by the homomorphism $\varphi: A \rightarrow \mathscr{B}\left(A \otimes l^{2} \mathbb{F}_{2}\right)$.

In particular, the bimodule is in fact the same as the bimodule appearing in the Julg and Valette cycle appearing in Lemma 7.3.

It remains to calculate a lift $\bar{U}$ of $\tau(v-\chi)$ and show that in fact such a lift can be chosen, which agrees with the operator $\bar{W}$ of Lemma 7.3.

We first construct a lift of $\tau(v)$. Recall that $v=\sum_{\gamma \in S} \chi_{\gamma} \gamma \otimes\left(1-\chi_{\gamma}\right) \gamma$, where $S$ is a basis for $\mathbb{F}_{2}$. Each $\gamma$ is mapped under $\tau$ to the image in the Calkin algebra 
of the right translation operators $1 \otimes \rho(\gamma): A \otimes l^{2} \mathbb{F}_{2} \rightarrow A \otimes l^{2} \mathbb{F}_{2}$. Consider each term $F_{\gamma}=\chi_{\gamma} \otimes\left(1-\chi_{\gamma}\right) \in C_{c}\left(\partial^{2} \mathbb{F}_{2}\right)$. Let $\tilde{\chi}_{\gamma}$ denote the function on $\mathbb{F}_{2}$ given by

$$
\tilde{X}_{\gamma}(g)= \begin{cases}1, & \gamma \in[e, g] \\ 0, & \text { else. }\end{cases}
$$

Then $\tilde{X}_{y}$ extends continuously to $\overline{\mathbb{F}}_{2}$, and the restriction of $\tilde{\chi}_{y}$ to $\partial \mathbb{F}_{2}$ is $\chi_{\gamma}$. Let then

$$
\tilde{F}_{\gamma}=\chi_{\gamma} \otimes\left(1-\tilde{X}_{y}\right)
$$

which is an extension to $\partial \mathbb{F}_{2} \times \overline{\mathbb{F}}_{2}$ of $F_{\gamma}$. Forming $\tilde{F}_{\gamma}^{\prime}$ as per the recipe described in the definition of $\tau$, we obtain the function

$$
\tilde{F}_{\gamma}^{\prime}(a, g)=\tilde{F}_{\gamma}\left(g^{-1} a, g^{-1}\right)= \begin{cases}1, & \gamma \in\left[e, g^{-1} a\right), \gamma \notin\left[e, g^{-1}\right], \\ 0, & \text { else. }\end{cases}
$$

We remind the reader that the statement " $x \in[e, y]$ " for $x, y \in \mathbb{F}_{2}$ may be equivalently read: "the reduced expression of $y$ contains $x$ as an initial subword," or more shortly, " $y$ begins with $x$."

With this in mind, consider the first case above. If $g^{-1} a$ begins with $\gamma$ but $g^{-1}$ does not, it follows that there is cancellation between $g^{-1}$ and $a$; more precisely, $a$ must begin with $g$, followed by $\gamma$. (Since $g^{-1}$ does not begin with $\gamma, g$ does not end in $\gamma^{-1}$, and hence $g \gamma$ is in fact reduced.) We have

$$
\tilde{F}_{\gamma}^{\prime}(a, g)= \begin{cases}1, & g \gamma \in[e, a), g \text { does not end in } \gamma^{-1}, \\ 0, & \text { else. }\end{cases}
$$

Now, consider the operator $\tilde{F}_{\gamma}^{\prime}\left(1 \otimes v_{\gamma}\right) \in A \otimes \mathscr{B}\left(l^{2} \mathbb{F}_{2}\right) \subset \mathscr{B}\left(A \otimes l^{2} \mathbb{F}_{2}\right)$. This operates by

$$
\left(\sum f_{h} h\right) \otimes e_{g} \longmapsto\left(\sum \tilde{F}_{\gamma}^{\prime}\left(\cdot, g \gamma^{-1}\right) f_{h} h\right) \otimes e_{g \gamma^{-1}}
$$

for $\sum f_{h} h$ is an arbitrary element of the cross product $A$. From our above work, we see that $\tilde{F}_{\gamma}^{\prime}\left(\cdot, g \gamma^{-1}\right)=0$ unless $g$ ends in $\gamma$. On the other hand, if $g$ does end in $\gamma, g \gamma^{-1}$ does not end in $\gamma^{-1}$. Hence, we see that the above operator sends

$$
\left(\sum f_{h} h\right) \otimes e_{g} \longmapsto \begin{cases}\left(\sum x_{g} f_{h} h\right) \otimes e_{g \gamma^{-1}}, & g \text { ends in } \gamma, \\ 0, & \text { else. }\end{cases}
$$

We see finally that $\bar{V}=\sum_{\gamma \in S} \tilde{F}_{\gamma}^{\prime} \cdot\left(1 \otimes v_{\gamma}\right)$, which is a lift of $\tau(v)$, acts on $A \otimes l^{2} \mathbb{F}_{2}$ by

$$
\left(\sum f_{h} h\right) \otimes e_{g} \longmapsto\left(\sum x_{g} f_{h} h\right) \otimes e_{g^{\prime}}
$$

where the prime notation is as in the discussion just prior to Lemma 7.2. 
In particular, $\bar{V}$ as an operator on $A \otimes l^{2} \mathbb{F}_{2}$, where the latter is regarded as functions $\mathbb{F}_{2} \rightarrow C\left(\partial \mathbb{F}_{2}\right) \otimes l^{2} \mathbb{F}_{2}$, has the form

$$
(\bar{V} \xi)(g)=V(\xi(g))
$$

where $V$ is the operator $C\left(\partial \mathbb{F}_{2}\right) \otimes l^{2} \mathbb{F}_{2} \rightarrow C\left(\partial \mathbb{F}_{2}\right) \otimes l^{2} \mathbb{F}_{2}$, where

$$
V\left(f \otimes e_{g}\right)=\chi_{g} f \otimes e_{g^{\prime}} .
$$

Otherwise expressed, let $\xi$ be an element of $C\left(\partial \mathbb{F}_{2} ; l^{2} \mathbb{F}_{2}\right)$ of the form $\xi(a)=$ $\sum \xi_{g}(a) e_{g}$, where each $\xi_{g}$ is a scalar-valued function on $\partial \mathbb{F}_{2}$. Then

$$
(V \xi)(a)=\sum_{g \in[e, a)} \xi_{g}(a) \otimes e_{g^{\prime}} .
$$

Now, apply the same calculations to the element $\tau(\chi)$. We obtain the operator (projection) $\bar{P}$ on $A \otimes l^{2} \mathbb{F}_{2}$ given by $\bar{P}=\sum \tilde{F}_{\gamma}^{\prime} \in A \otimes \mathscr{B}\left(l^{2} \mathbb{F}_{2}\right) \subset \mathscr{B}\left(A \otimes l^{2} \mathbb{F}_{2}\right)$.

We have that $\bar{V}-\bar{P}$ is an operator whose projection to the Calkin algebra is $\tau(v-\chi)$ as required. Let it be denoted by $\bar{U}$. Form $\bar{F}=\bar{U}+1$.

Our calculations show that $\bar{F}$ is an operator having the form

$$
(\bar{F} \xi)(g)=F(\xi(g)),
$$

where $F: C\left(\partial \mathbb{F}_{2}\right) \otimes l^{2} \mathbb{F}_{2} \rightarrow C\left(\partial \mathbb{F}_{2}\right) \otimes l^{2} \mathbb{F}_{2}$ is the operator

$$
(F \xi)(a)=\sum_{g \in[e, a)} \xi_{g}(a) \otimes e_{g^{\prime}}+\sum_{g \notin[e, a)} x_{g}(a) \otimes e_{g},
$$

which is precisely the operator $W$ of Lemma 7.2, that is, $F=W$, and therefore $\bar{F}=\bar{W} \in \mathscr{B}\left(A \otimes l^{2} \mathbb{F}_{2}\right)$.

We are now done, having shown by direct computation that

$$
\begin{aligned}
\left([D] \otimes 1_{A}\right) \otimes_{B \otimes A}\left[i \otimes 1_{A}\right] \otimes_{A \otimes A \otimes A}\left(1_{A} \otimes \sigma_{12}^{*} \Delta\right) & \\
= & {\left[\left(C\left(\partial \mathbb{F}_{2}\right) \rtimes \mathbb{F}_{2} \otimes l^{2} \mathbb{F}_{2}, \bar{W}\right)\right]=\lambda\left(\gamma_{\partial \mathbb{F}_{2} \rtimes \mathbb{F}_{2}}\right), }
\end{aligned}
$$

and therefore that

$$
\left([D] \otimes 1_{A}\right) \otimes_{B \otimes A}\left[i \otimes 1_{A}\right] \otimes_{A \otimes A \otimes A}\left(1_{A} \otimes \sigma_{12}^{*} \Delta\right)=1_{A} .
$$

\section{REFERENCES}

[1] C. Anantharaman-Delaroche and J. Renault, Amenable Groupoids, Monographie de L'Enseignement Mathématique, vol. 36, L’Enseignement Mathématique, Geneva, 2000.

[2] B. Blackadar, K-Theory for Operator Algebras, Mathematical Sciences Research Institute Publications, vol. 5, Springer-Verlag, New York, 1986.

[3] A. Connes, Noncommutative Geometry, Academic Press, California, 1996. 
[4] E. Ghys and P. de la Harpe (eds.), Sur les Groupes Hyperboliques d'après Mikhael Gromov [Hyperbolic Groups in the Theory of Mikhael Gromov], Progress in Mathematics, vol. 83, Birkhäuser Boston, Massachusetts, 1990 (French).

[5] M. Gromov, Hyperbolic groups, Essays in Group Theory, Math. Sci. Res. Inst. Publ., vol. 8, Springer, New York, 1987, pp. 75-263.

[6] N. Higson, A characterization of KK-theory, Pacific J. Math. 126 (1987), no. 2, 253-276.

[7] P. Julg and A. Valette, $K$-theoretic amenability for $\mathrm{SL}_{2}\left(\mathbf{Q}_{p}\right)$, and the action on the associated tree, J. Funct. Anal. 58 (1984), no. 2, 194-215.

[8] J. Kaminker and I. Putnam, $K$-theoretic duality of shifts of finite type, Comm. Math. Phys. 187 (1997), no. 3, 509-522.

[9] G. G. Kasparov, Equivariant KK-theory and the Novikov conjecture, Invent. Math. 91 (1988), no. 1, 147-201.

[10] M. A. Rieffel, Applications of strong Morita equivalence to transformation group $C^{*}$-algebras, Operator Algebras and Applications, Part I (Kingston, Ont., 1980), Proc. Sympos. Pure Math., vol. 38, American Mathematical Society, Rhode Island, 1982, pp. 299-310.

[11] J.-L. Tu, La Conjecture de Baum-Connes pour les Feuilletages Moyennables [The Baum-Connes conjecture for amenable foliations], K-Theory 17 (1999), no. 3, 215-264 (French).

Heath Emerson: Indiana University-Purdue University at Indianapolis (IUPUI), 402 North Blackford Street, Indianapolis, IN 46202-3216, USA

Current address: Mathematisches Institut, Westfalische Wilhelms-Universitat, Einsteinstrasse 62, 48149 Muenster, Germany

E-mail address: hemerson@math . iupui . edu 


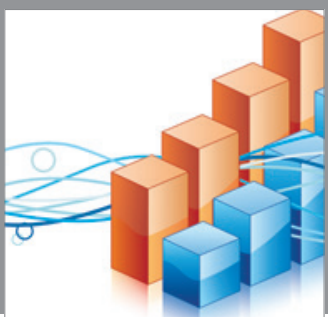

Advances in

Operations Research

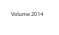

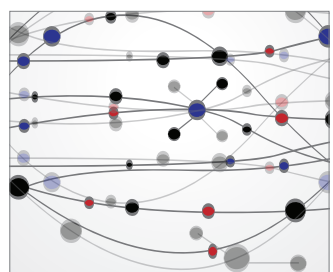

\section{The Scientific} World Journal
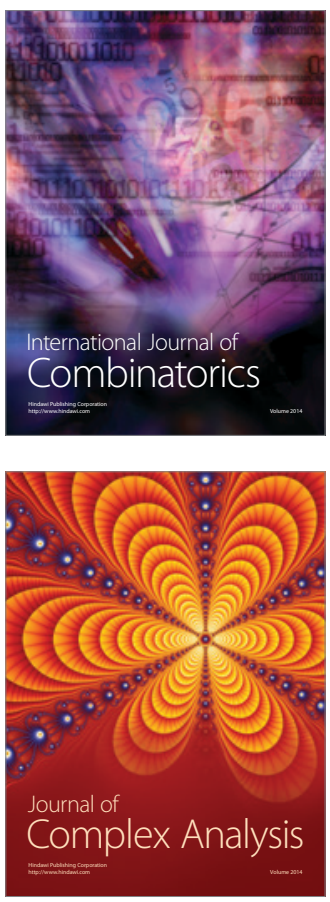

International Journal of

Mathematics and

Mathematical

Sciences
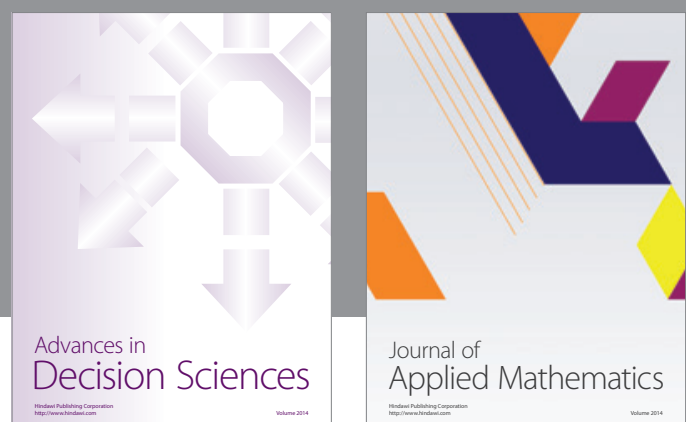

Journal of

Applied Mathematics
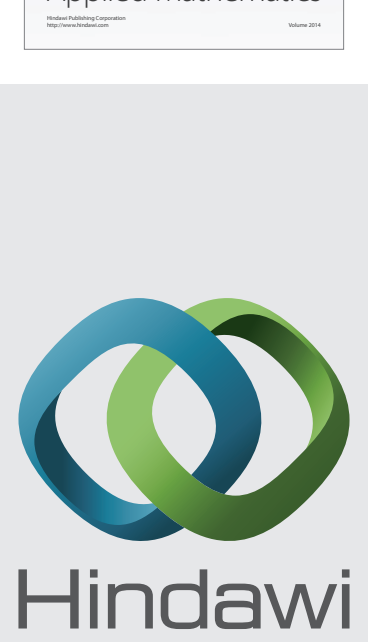

Submit your manuscripts at http://www.hindawi.com
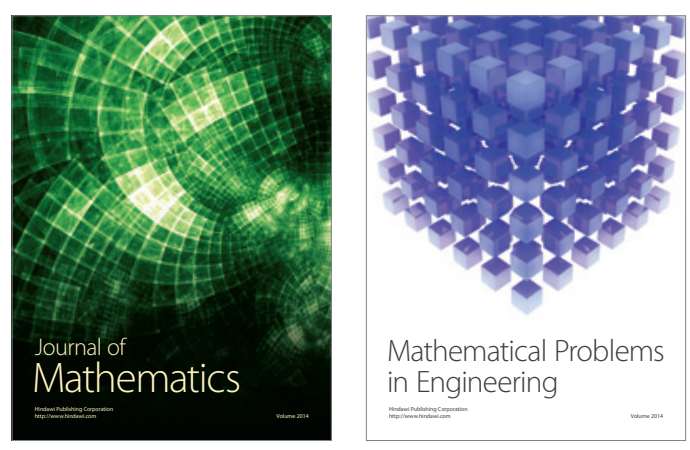

Mathematical Problems in Engineering
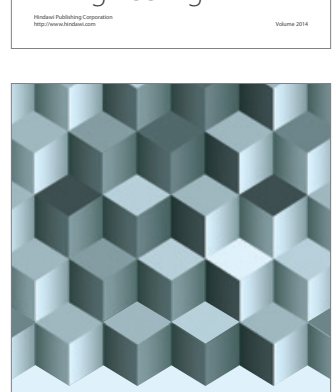

Journal of

Function Spaces
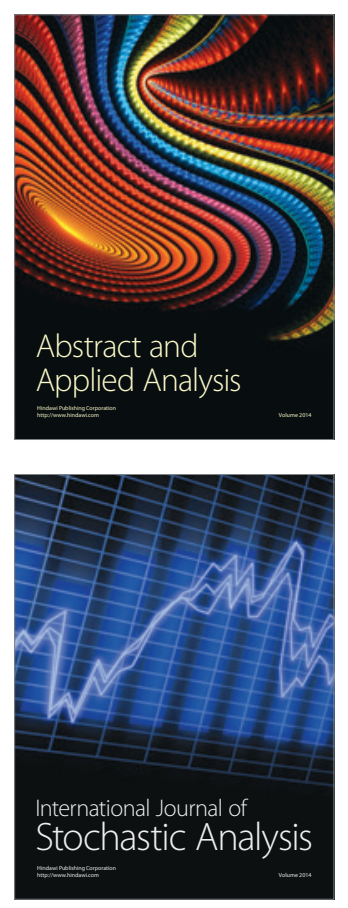

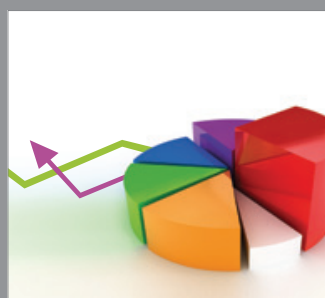

ournal of

Probability and Statistics

Promensencen
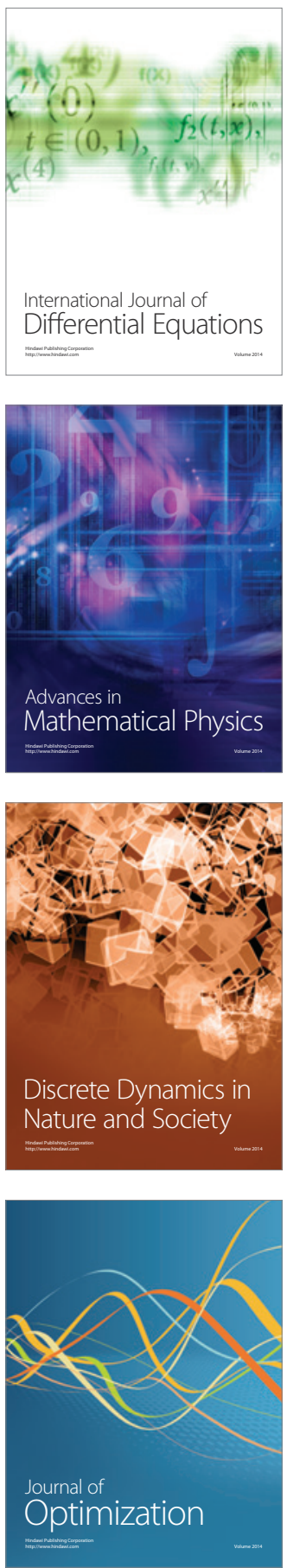